\title{
Clinical Usefulness of Cancer Antigen (CA) 125, Human Epididymis 4, and CA72-4 Levels and Risk of Ovarian Malignancy Algorithm Values for Diagnosing Ovarian Tumors in Korean Patients With and Without Endometriosis
}

\author{
Kyung-Hwa Shin (10, M.D., Ph.D. ${ }^{1}$, Hyung-Hoi Kim (1), M.D., Ph.D. ${ }^{1}$, Byung Su Kwon (®), M.D., Ph.D. ${ }^{2,3}$,
}

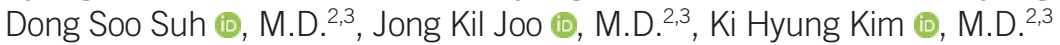

${ }^{1}$ Department of Laboratory Medicine and Biomedical Research Institute, Pusan National University Hospital and Pusan National University School of Medicine, Busan, Korea; ${ }^{2}$ Department of Obstetrics and Gynecology, Pusan National University School of Medicine, Busan, Korea; ${ }^{3}$ Biomedical Research Institute, Pusan National University Hospital, Busan, Korea

Background: Tumor markers are useful for detection and preoperative evaluation of ovarian tumors. We evaluated the clinical usefulness of cancer antigen (CA) 125, human epididymis 4 (HE4), and CA72-4 levels and Risk of Ovarian Malignancy Algorithm (ROMA) values for differential diagnosis of malignant and borderline tumors among suspected ovarian tumors, and the effects of endometriosis on these tumor markers.

Methods: In a total of 266 patients $(213,14$, and 39 with benign, borderline and malignant tumors, respectively), CA125, HE4, and CA72-4 levels were measured, and ROMA values were calculated. Medians of each marker were compared among the three groups. The area under the ROC curve (AUC), sensitivity, and specificity were calculated to analyze the diagnostic performance of each marker.

Results: All markers were significantly higher in the malignant group than in the benign group. HE4 levels and ROMA values were significantly higher in the malignant group than in the borderline group. ROMA value had the highest AUC for distinguishing the malignant and borderline groups from the benign group in premenopausal (0.773) and postmenopausal (0.927) patients. CA125 level was significantly higher in patients with endometriosis than in those without $(P<0.001)$, whereas HE4 and CA72-4 levels were not affected by endometriosis ( $P=0.128$ and 0.271 , respectively).

Conclusions: ROMA value is the best marker to distinguish malignant and borderline tumors from benign tumors in pre- and postmenopausal patients. HE4 and CA72-4 levels provide information on possible CA125 elevation due to endometriosis.

Key Words: Cancer antigen 125, Human epididymis 4, Cancer antigen 72-4, Risk of Ovarian Malignancy Algorithm, Ovarian tumor
Received: January 30, 2019

Revision received: April 10, 2019

Accepted: August 2, 2019

\section{Corresponding author:}

Hyung-Hoi Kim, M.D., Ph.D.

Department of Laboratory Medicine and and Biomedical Research Institute, Pusan National University Hospital and Pusan National University School of Medicine, 179 Gudeok-ro, Seo-gu, Busan 49241, Korea

Tel: +82-51-240-7414

Fax: +82-51-247-6560

E-mail: hhkim@pusan.ac.kr

\section{INTRODUCTION}

Ovarian cancer is the eighth most common cancer in women; 290,000 new ovarian cancer cases and 184,000 ovarian cancer- related deaths were estimated to occur in 2018 worldwide [1]. Early detection of ovarian cancer is associated with good prognosis, whereas the five-yr survival rate is very poor $(20 \%-40 \%)$ in patients diagnosed at advanced stages. However, only $20 \%$ of 
ovarian cancers are diagnosed at an early stage. Therefore, there is a need for effective detection of early-stage cancer, which requires screening tools with high sensitivity and specificity [1, 2].

Estimation of cancer antigen 125 (CA125) levels and transvaginal sonography are the best and most frequently recommended methods for diagnosing ovarian cancer [3-5]. However, abnormally high CA125 levels are detected in malignancies of different origins [6]. CA125 levels are strongly affected by various physiological factors (e.g., pregnancy and different phases of the menstrual cycle) and benign conditions, including endometriosis [7]. Therefore, new markers, including human epididymis 4 (HE4) and CA72-4, have been proposed and evaluated. HE4 is overexpressed in ovarian cancer tissues compared with normal ovarian tissues [8]. CA72-4 levels are increased in various epithelial cancers [9]. Although CA72-4 is less sensitive than CA125, it has the advantage of not being influenced by the menstrual cycle or pregnancy $[10,11]$. However, the usefulness of CA72-4 in differential diagnosis of ovarian cancer is not well known.

As no single marker is sufficient for diagnosing ovarian cancers, multiple tests or testing patterns can be combined to achieve high sensitivity and specificity. Potential serum markers that can be tested in combination include CA125, HE4, CA72-4, and TP53 autoantibodies [12]. For example, multiple markers are incorporated in the Risk of Ovarian Malignancy Algorithm (ROMA), Risk of Malignant Index, OVA1 test, and LR2 index [13].

To our knowledge, no study has evaluated the clinical usefulness of tumor markers including CA72-4 in Korean patients with ovarian tumors. We evaluated the clinical usefulness of CA125, HE4, and CA72-4 levels and ROMA values for differential diagnosis of malignant and borderline tumors among suspected ovarian tumors. We also examined the ability of a combination of tumor makers to improve diagnostic yield for malignant and borderline tumors. The effects of endometriosis on tumor markers were also evaluated.

\section{METHODS}

\section{Study design and population}

This was a retrospective and observational single-center study. Blood samples were collected from 354 patients who underwent a planned surgical procedure at the gynecology clinic at Pusan National University Hospital, Busan, Korea, for a symptomatic or suspected malignant ovarian tumor between June 2015 and July 2018. Samples were collected within one month before surgery; 88 patients were excluded because they underwent pre-operative testing but did not undergo surgery. The sera and clinical information used in this study were provided by the Biobank of Pusan National University Hospital, a member of the Korea Biobank Network. The study protocol was approved by the Institutional Review Board of Pusan National University Hospital (E-2015088). Informed consent for collection, storage, and use of blood samples in biobank was obtained from the patients.

Information on menopausal status, age, diagnosis, histological type of tumor, and day of surgery was collected; when information about menopausal status was not provided, patients $<50$ years were considered premenopausal, and patients $\geq 50$ years were considered postmenopausal. Two pathologists confirmed the status of surgically resected ovarian tumor tissue histologically, using the International Federation of Gynecology and Obstetrics (FIGO) ovarian staging classification [14].

Two hundred and sixty-six patients $(213,14$, and 39 with benign, borderline, and malignant tumors, respectively) were enrolled. The median age (range) at initial diagnosis was 40 years (13-80 years), 42.5 years (13-79 years), and 51 years (13-78 years) for the benign, borderline, and malignant groups, respectively. In total, 177 premenopausal and 89 postmenopausal patients were enrolled. Among the benign group patients, 150 were premenopausal, and 63 were postmenopausal. In the borderline and malignant groups, eight and 19 patients were premenopausal, respectively, whereas six and 20 patients were postmenopausal, respectively. The histological types of the ovarian tumor are shown in Table 1. In the malignant group, there were 15 patients with FIGO stage I, four with stage II, 14 with stage III, and six patients with stage IV.

\section{Testing of tumor markers}

Blood samples were collected in serum separator tubes, centrifuged within 30 minutes after arrival at the laboratory, and stored at $-70^{\circ} \mathrm{C}$ before use. After the samples were thawed, the markers were measured simultaneously. CA125, HE4, and CA72-4 were measured using Elecsys electrochemiluminescence immunoassays (Roche Diagnostics, Basel, Switzerland) for in the cobas e602 analyzer (Roche Diagnostics). The measurable ranges were $0.6-5,000 \mathrm{U} / \mathrm{mL}$ for CA125, 15-1,500 pmol/L for HE4, and $0.2-300 \mathrm{U} / \mathrm{mL}$ for CA72-4.

ROMA values for pre- and postmenopausal patients were calculated based on CA125 and HE4 assay results. The ROMA value (\%) was calculated as:

$\exp (\mathrm{PI}) /[1+\exp (\mathrm{PI}) \times 100]$, where $\exp (\mathrm{PI})=\mathrm{e}^{\mathrm{PI}}$ and $\mathrm{PI}$ is the calculated predictive value [20].

The PI was calculated for pre- and postmenopausal patients separately; PI (premenopausal) was calculated as $-12.0+2.38$ 
Table 1. Histopathologic types of tumor in patients with ovarian tumors enrolled for differential diagnosis of malignant tumor $(\mathrm{N}=266)$

\begin{tabular}{|c|c|c|c|}
\hline & & Histopathologic type & $\mathrm{N}$ \\
\hline \multirow[t]{11}{*}{ Benign $(N=213)$} & Non-neoplastic mass & Endometrioma & 39 \\
\hline & & Simple or follicular cyst & 9 \\
\hline & & Corpus luteal cyst with/without endometriosis & 12 \\
\hline & & Paratubal cyst with/without endometriosis & 16 \\
\hline & & Other* & 21 \\
\hline & Epithelial ovarian tumor & Mucinous & 32 \\
\hline & & Serous & 18 \\
\hline & & Mixed & 2 \\
\hline & Germ cell tumor & Mature cystic teratoma & 56 \\
\hline & Sex cord-stromal tumor & Thecoma-fibroma & 7 \\
\hline & & Leydig cell & 1 \\
\hline \multirow[t]{3}{*}{ Borderline $(\mathrm{N}=14)$} & Epithelial ovarian tumor & Mucinous & 10 \\
\hline & & Serous & 3 \\
\hline & & Mixed & 1 \\
\hline \multirow[t]{9}{*}{ Malignant $(\mathrm{N}=39)$} & Epithelial ovarian tumor & Mucinous cystadenocarcinoma & 5 \\
\hline & & Serous & 14 \\
\hline & & Endometrioid & 2 \\
\hline & & Clear cell & 5 \\
\hline & & Mixed & 1 \\
\hline & Germ cell tumor & Dysgerminoma & 1 \\
\hline & & Endodermal sinus & 1 \\
\hline & Sex cord stromal tumor & Granulosa cell & 2 \\
\hline & Metastatic cancer & & 8 \\
\hline
\end{tabular}

* "Other" includes no tumor in ovary $(\mathrm{N}=16)$, salpingo-oophoritis $(\mathrm{N}=1)$, vascular congestion $(\mathrm{N}=1)$, hydrosalpinx $(\mathrm{N}=1)$, chronic granulomatous inflammation ( $\mathrm{N}=1)$, and cortical inclusion cyst $(\mathrm{N}=1)$.

$\times$ LN[HE4]+0.0626 $\times$ LN[CA125], and PI (postmenopausal) was calculated as $-8.09+1.04 \times$ LN[HE4]+0.732 $\times$ LN[CA125] [15].

Recommended cut-off levels were CA125 $\geq 35 \mathrm{U} / \mathrm{mL}$, HE4 (premenopausal) $\geq 92.1 \mathrm{pmol} / \mathrm{L}, \mathrm{HE} 4$ (postmenopausal) $\geq 121$ pmol/L, CA72-4 >6.9 U/mL, ROMA (premenopausal) value $\geq 11.4 \%$, and ROMA (postmenopausal) value $\geq 29.9 \%$, according to the manufacturer's instructions.

\section{Statistical analysis}

Continuous variables were expressed as medians and interquartile ranges (IQRs). Significant differences in parameters among groups were evaluated using the Kruskal-Wallis test for threegroup comparisons, and the Mann-Whitney U-test for two-group comparisons. Box plots were constructed to represent marker levels (displaying with the middle two, range and median). The outlier are data points that more than lower quartile $-1.5 \times I Q R$ or upper quartile+1.5 $\times \mathrm{IQR}$ and extreme are data posint that more extreme than lower quartile $-3 \times \mid Q R$ or upper quartile $+3 \times I Q R$. To analyze the diagnostic performance of each tumor marker and the ROMA value, sensitivity and specificity were calculated, ROC curves were constructed, and the area under the curve (AUC) of each marker was calculated.

All analyses were performed with SPSS version 23 (IBM Corp, Armonk, NY, USA). $P<0.05$ (for two-group comparisons) and $P<0.017$ (for three-group comparisons) were considered significant. Sensitivity and specificity for differential diagnosis of patients in the malignant and borderline groups were calculated for single and various combinations of tumor markers.

\section{RESULTS}

Tumor markers for differential diagnosis of malignant tumor For each group, CA125, HE4, and CA72-4 levels and ROMA values were estimated (Table 2). In premenopausal patients, HE4 
Table 2. Serum marker levels and ROMA values according to menopausal status

\begin{tabular}{lcccc}
\hline & Benign & Borderline & Malignant & $P^{*}$ \\
\hline Total (N) & 213 & 14 & 39 & \\
CA125 (U/mL) & $18.6(12.5-33.0)^{\dagger}$ & $37.0(16.8-144.6)$ & $138.1(18.6-719.7)$ & $<0.001$ \\
HE4 (pmol/L) & $46.9(39.9-54.8)^{\dagger}$ & $54.5(46.3-80.4)^{\ddagger}$ & $139.6(63.0-488.0)$ & $<0.001$ \\
CA72-4 (U/mL) & $1.3(0.9-2.4)^{\dagger}$ & $2.1(1.1-4.0)$ & $3.0(1.2-7.9)$ & 0.001 \\
ROMA (premenopausal) (\%)" & $6.4(4.6-9.5)^{\dagger}$ & $9.7(6.4-20.1)^{\ddagger}$ & $49.1(12.8-95.5)$ & $<0.001$ \\
ROMA (postmenopausal) (\%)" & $13.5(9.1-19.6)^{\dagger}$ & $21.2(17.0-39.7)^{\ddagger}, 8$ & $63.3(31.8-93.0)$ & $<0.001$ \\
Premenopausal (N) & 150 & 8 & 19 & \\
CA125 (U/mL) & $22.1(15.0-37.7)$ & $51.4(18.9-146.1)$ & $48.5(12.7-136.2)$ & 0.12 \\
HE4 (pmol/L) & $43.9(39.3-51.2)^{\dagger}$ & $51.6(47.3-71.5)$ & $67.5(47.9-146.8)$ & $<0.001$ \\
CA72-4 (U/mL) & $1.3(0.9-2.4)$ & $1.1(1.0-2.9)$ & $1.5(0.8-5.8)$ & 0.751 \\
ROMA (\%) & $5.7(4.4-8.4)^{\dagger}$ & $9.1(7.2-16.0)$ & $13.9(6.5-51.4)$ & $<0.001$ \\
Postmenopausal (N) & 63 & 6 & 20 & $<0.001$ \\
CA125 (U/mL) & $13.1(9.7-17.7)^{\dagger}$ & $17.0(12.7-140.1)^{\ddagger}$ & $377.0(122.1-959.2)$ & $<0.001$ \\
HE4 (pmol/L) & $54.3(46.5-72.7)^{\dagger}$ & $71.3(45.9-83.4)^{\ddagger}$ & $227.4(124.8-525.7)$ & $<0.001$ \\
CA72-4 (U/mL) & $1.1(0.8-2.18)^{\dagger}$ & $2.6(2.2-8.6)$ & $4.7(2.2-11.2)$ & $<0.001$ \\
ROMA (\%) & $12.1(8.8-18.0)^{\dagger}$ & $18.3(11.4-34.3)^{\ddagger}$ & $90.5(65.2-95.0)$ & \\
\hline
\end{tabular}

Values are expressed as medians and interquartile ranges.

${ }^{*}$ Indicates a significant difference between malignant, borderline, and benign groups by the Kruskal-Wallis test $(P<0.017)$; ${ }^{\dagger}$ Malignant group vs benign group $(P<0.05)$; ${ }^{\ddagger}$ Malignant group vs borderline group $(P<0.05)$; ${ }^{\S}$ Borderline group vs benign group $(P<0.05)$; "Results included patients in premenopausal and postmenopausal state.

Abbreviations: CA, cancer antigen; HE4, human epididymis 4; ROMA, Risk of Ovarian Malignancy Algorithm.

levels and ROMA values were higher in the malignant group than in the benign group $(P<0.001)$. In postmenopausal patients, CA125 and HE4 levels and ROMA values were higher in the malignant group than in the borderline $(P=0.009, P=0.002$, and $P=0.001$, respectively) and benign (all $P<0.001$ ) groups. CA72-4 levels were higher in the malignant group than in the benign group $(P<0.001)$.

Among the 19 premenopausal patients in the malignant group, four patients showed high CA125 and HE4 levels; six patients showed high CA125 levels; two patients showed high HE4 levels; and 10 patients showed high ROMA values ( $\geq 11.4 \%$ ). The seven patients with CA125 and HE4 levels below the reference range were patients with mucinous carcinoma, metastatic carcinoma, granulosa cell tumor, or low-grade serous tumor. The two patients with only high HE4 level had endometrioid-type carcinoma. Among the five patients who showed a high CA72-4 level, two showed high CA125 and HE4 levels, two showed only a high CA125 level, and one patient showed CA125 and HE4 levels below the reference range. Among the 20 postmenopausal patients in the malignant group, 14 showed both high CA125 and HE4 levels, four showed only high CA125 level, one showed only high HE4 level, and 19 patients showed a high ROMA value
( $\geq 29.9 \%$ ). One patient with CA125 and HE4 levels below the reference range had clear-cell carcinoma. Among the eight patients with a high CA72-4 level, six showed high CA125 and HE4 levels, and two showed only a high CA125 level.

Among the eight premenopausal patients in the borderline group, one showed both high CA125 and HE4 levels and a high ROMA value ( $\geq 11.4 \%$ ), whereas four showed only a high CA125 level and one only a high HE4 level, respectively. Two patients showed CA125 and HE4 levels below the reference range, whereas there were no patients with a high CA72-4 level. Among the six postmenopausal patients in the borderline group, one showed both high CA125 and HE4 levels and a high ROMA value ( $\geq 29.9 \%$ ), whereas one patient showed only a high CA125 level. Four patients showed CA125 and HE4 levels below the reference range. Among the two patients showing high CA72-4 level, one patient showed high CA125 and HE4 levels, and one patient showed CA125 and HE4 levels below the reference range.

Sensitivity, specificity, and AUC values for distinguishing between malignant/borderline and benign groups and between malignant and borderline/benign groups are shown in Table 3 . ROMA value showed the highest sensitivity among all markers, and CA125 showed the highest sensitivity as a single marker. 
Table 3. Sensitivity and specificity of combinations of tumor markers in predicting malignant tumor/borderline tumor and malignant tumor only

\begin{tabular}{|c|c|c|c|c|c|c|c|}
\hline \multirow{2}{*}{ Menopausal status } & \multirow{2}{*}{$\begin{array}{l}\text { Combination of } \\
\text { tumor markers }\end{array}$} & \multicolumn{3}{|c|}{ Malignant+borderline vs benign } & \multicolumn{3}{|c|}{ Malignant vs borderline+benign } \\
\hline & & Sensitivity (\%) & Specificity (\%) & AUC & Sensitivity (\%) & Specificity (\%) & AUC \\
\hline \multirow[t]{9}{*}{ Premenopausal } & CA125 & 53.6 & 71.6 & 0.603 & 52.6 & 70.1 & 0.569 \\
\hline & HE4 & 28.6 & 98.6 & 0.767 & 31.6 & 97.5 & 0.793 \\
\hline & CA72-4 & 17.9 & 92.6 & 0.528 & 26.3 & 93.0 & 0.552 \\
\hline & ROMA & 46.4 & 89.2 & 0.773 & 52.6 & 87.9 & 0.792 \\
\hline & CA125/HE4 & 66.7 & 70.7 & NA & 63.2 & 68.3 & NA \\
\hline & CA125/CA72-4 & 66.7 & 70.7 & NA & 63.2 & 68.3 & NA \\
\hline & HE4/CA72-4 & 40.7 & 91.3 & NA & 47.4 & 90.5 & NA \\
\hline & ROMA/CA72-4 & 44.4 & 82.0 & NA & 52.6 & 81.6 & NA \\
\hline & CA125/HE4/CA72-4 & 70.4 & 64.7 & NA & 68.4 & 62.7 & NA \\
\hline \multirow[t]{9}{*}{ Postmenopausal } & CA125 & 80.0 & 87.7 & 0.870 & 90.0 & 85.7 & 0.917 \\
\hline & HE4 & 64.0 & 92.3 & 0.865 & 75.0 & 91.4 & 0.939 \\
\hline & CA72-4 & 40.0 & 98.5 & 0.820 & 40.0 & 95.7 & 0.787 \\
\hline & ROMA & 84.0 & 89.2 & 0.927 & 95.0 & 87.1 & 0.980 \\
\hline & CA125/HE4 & 80.8 & 80.9 & NA & 95.0 & 79.7 & NA \\
\hline & CA125/CA72-4 & 80.8 & 85.7 & $N A$ & 90.0 & 82.6 & NA \\
\hline & HE4/CA72-4 & 79.2 & 90.5 & NA & 85.0 & 88.4 & NA \\
\hline & ROMA/CA72-4 & 92.3 & 60.3 & $N A$ & 100.0 & 58.0 & NA \\
\hline & CA125/HE4/CA72-4 & 80.8 & 85.7 & NA & 90.0 & 82.6 & NA \\
\hline
\end{tabular}

Abbreviations: AUC, area under the ROC curve; CA, cancer antigen; HE4, human epididymis 4; ROMA, Risk of Ovarian Malignancy Algorithm, NA, not available.

HE4 and CA72-4 showed higher specificity than CA125 and ROMA value. The sensitivities of all markers were higher in postmenopausal than in premenopausal patients. The combination of HE4 or CA72-4 with CA125 showed higher sensitivity than CA125 alone in premenopausal patients. The combination of CA125, HE4, and CA72-4 did not improve sensitivity and specificity compared with the combination of HE4 or CA72-4 with CA125.

\section{Effect of endometriosis on tumor markers}

Among premenopausal patients, 53 (35.6\%) had endometriosis (confirmed by histological examination), and among postmenopausal patients, six (9.5\%) had endometriosis. In total, 42.3\% (25/59) of the patients with endometriosis had high CA125 levels, whereas only one (1.7\%) and two (3.4\%) had high HE4 and CA72-4 levels, respectively. Six premenopausal patients (11.3\%) with endometriosis had a high ROMA value ( $\geq 11.4 \%$ ), whereas no postmenopausal patient with endometriosis had a high ROMA value ( $\geq 29.9 \%$ ).

As shown in Fig. 1, in the benign group, the CA125 level (median 32.4 U/mL, IQR 20.2-60.6 U/mL) was higher in patients with endometriosis than in those without endometriosis (median $15.3 \mathrm{U} / \mathrm{mL}$, IQR 11.2-25.9 U/mL) $(P<0.001)$; HE4 and CA72-4 levels were not affected by endometriosis. For HE4 and CA72-4, median values of $45.6 \mathrm{pmol} / \mathrm{L}$ (IQR 39.3-51.7 pmol/L) and 1.4 $\mathrm{U} / \mathrm{mL}$ (IQR 1.0-2.3 U/mL) were observed in patients with endometriosis, and median values of $46.9 \mathrm{pmol} / \mathrm{L}$ (IQR 40.2-57.3 pmol/L) and $1.2 \mathrm{U} / \mathrm{mL}$ (IQR 0.9-2.6 U/mL) were observed in patients without endometriosis $(P=0.128$ and 0.271$)$. The ROMA (postmenopausal) value was higher in patients with endometriosis (median 16.5\% [IQR 13.6-26.0\%]) than in patients without endometriosis (median 12.1\% [IQR 8.9-17.3\%]; $P<0.001$ ), whereas the ROMA (premenopausal) value did not differ (median 6.2\% [IQR 4.5-8.6\%] vs 6.6\% [4.6-10.0\%]) between the patients with and without endometriosis $(P=0.312)$.

\section{DISCUSSION}

The present study evaluated the clinical usefulness of CA125, HE4, and CA72-4 levels and ROMA values for differential diagnosis of malignant and borderline tumors among suspected ovarian tumors. CA125 showed the highest sensitivity, and HE4 

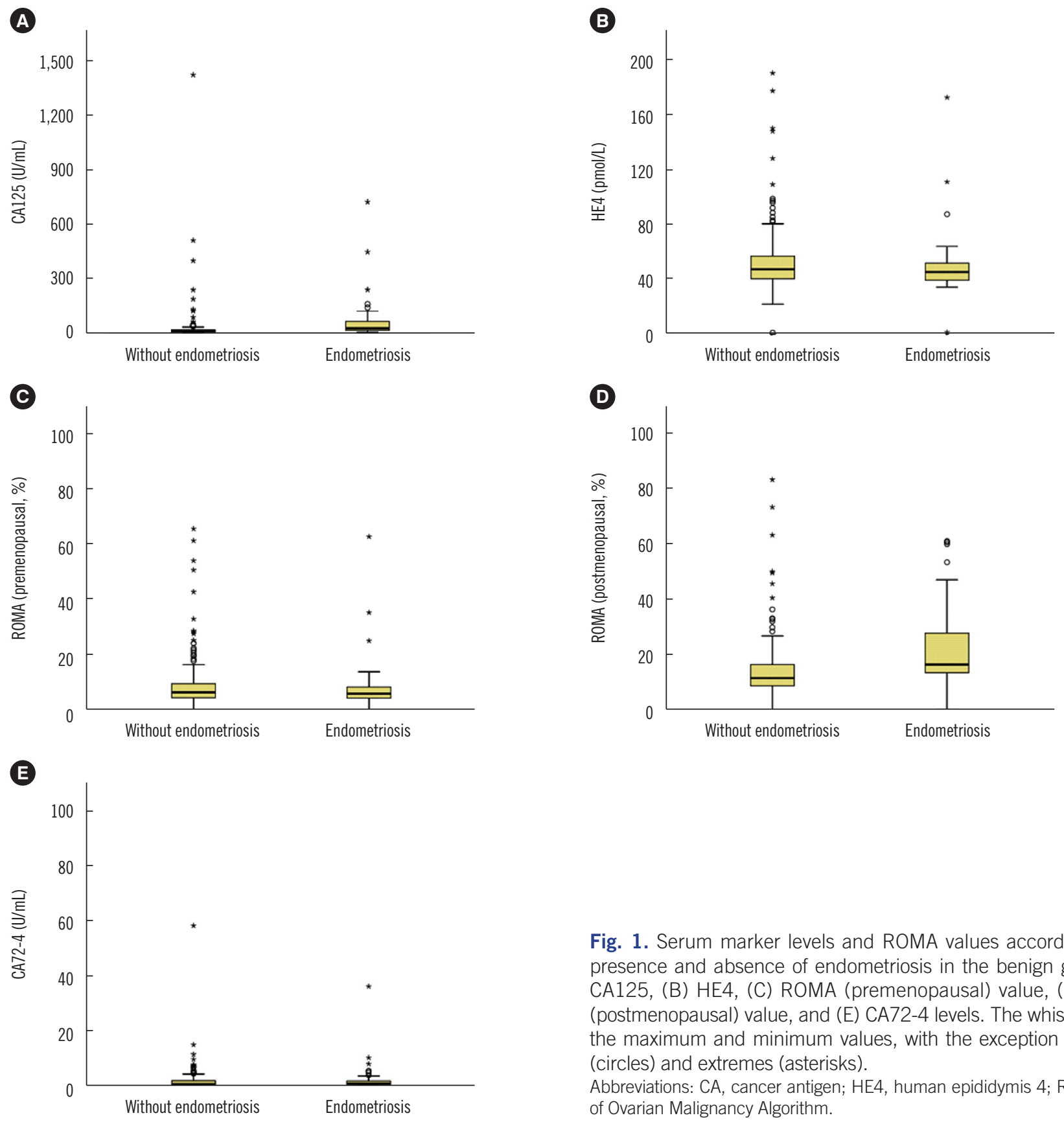

showed higher specificity for diagnosing malignant tumor using the recommended cut-off in premenopausal and postmenopausal patients. CA125 has a high sensitivity, but poor specificity, which often does not exceed $40 \%$ [13]. A meta-analysis of 16 datasets covering 1,342 ovarian cancer patients and 2,516 controls revealed an overall sensitivity of $79 \%$ and specificity of 93\% for HE4 and overall sensitivity of $79 \%$ and specificity of $78 \%$ for CA125 [16]. Another meta-analysis based on 28 studies indicated that the sensitivity of CA125 and HE4 levels and
Fig. 1. Serum marker levels and ROMA values according to the presence and absence of endometriosis in the benign group. (A) CA125, (B) HE4, (C) ROMA (premenopausal) value, (D) ROMA (postmenopausal) value, and (E) CA72-4 levels. The whiskers show the maximum and minimum values, with the exception of outliers (circles) and extremes (asterisks).

Abbreviations: CA, cancer antigen; HE4, human epididymis 4; ROMA, Risk of Ovarian Malignancy Algorithm.

ROMA values for diagnosing ovarian cancer was $76.3 \%, 79.2 \%$, and $85.3 \%$, respectively, and the specificity was $96.6 \%, 82.1 \%$, and $82.4 \%$, respectively [17]. The significantly higher specificity of HE4 than that of CA125 indicates that HE4 is less likely to be affected by factors other than the malignancy itself. Moreover, HE4 showed better results than CA125 for monitoring ovarian cancer [18]. Numerous reports indicated that combining CA125 with other laboratory and imaging results significantly improved specificity [15, 19-22]. 
HE4 has been shown to be the most useful marker for differential diagnosis of ovarian cancer and endometriosis [23, 24]. In our study, only $1.7 \%$ and $3.4 \%$ of patients with endometriosis showed increased HE4 and CA72-4 levels, respectively. In previous studies, $42.3 \%$ of women with endometriosis had high CA125 levels, confirming the low specificity of this marker [25, 26]. In our study, CA125 level was higher in patients with endometriosis than in patients without endometriosis, whereas HE4 and CA72-4 levels were not influenced by endometriosis. The role of CA72-4 in differential diagnosis of benign and malignant ovarian tumors is controversial. CA72-4 levels differed between women with ovarian cancer and those with endometriosis [25]. CA72-4 level was elevated in ovarian cancer with limited sensitivity as a single marker; however, when CA72-4 was combined with CA125 and HE4, the sensitivity of the triple marker assay increased by $2.4 \%$ compared with that of the dual marker combination of CA125 and HE4 [26]. In our study, of the 53 patients with malignant and borderline tumors, two patients had only high CA72-4 levels. Furthermore, high CA72-4 levels were found in two among the 59 patients with endometriosis and in 10 among the 154 patients with benign tumor without endometriosis.

ROMA value showed a better AUC value and sensitivity than CA125, HE4, and CA72-4 levels in pre- and postmenopausal patients in the present study. A previous study reported that ROMA value showed better performance than CA125 alone or HE4 alone [13]. However, other reports contradicted this finding [27-29]. Chan, et al. [28] and Kadija, et al. [29] demonstrated that assessment of ROMA value might be important for improving diagnostic performance in premenopausal patients, as it differentiates malignant tumors from endometrial cysts in premenopausal women.

A limitation of this study is the insufficient number of malignant and borderline tumors. Consequently, it was not possible to analyze diagnostic performance of each marker for differential diagnosis of malignant and borderline ovarian tumor according to various histologic subtypes. Because ovarian tumor has multiple histologic subtypes and the effect of each marker is different, multicenter studies are needed to clarify the histologic subtype correlated with the change in each marker for diagnosis, treatment, and prognosis of ovarian tumors.

In conclusion, ROMA value was the best marker to distinguish malignant and borderline tumors from benign tumors in both preand postmenopausal patients. HE4 and CA72-4 can be used in combination with CA125 to increase the diagnostic sensitivity in premenopausal patients. HE4 and CA72-4 provide information on the possibility of CA125 elevation by endometriosis.

\section{Acknowledgments}

All reagents were provided by Roche.

\section{Author Contributions}

All authors have accepted their responsibility for the entire content of this manuscript and approved submission. Kyung-Hwa Shin conducted the study and analyzed the data. Hyung-Hoi Kim organized the study and is the corresponding author. Byung Su Kwon, Dong Soo Suh, Jong Kil Joo, and Ki Hyung Kim collected the data.

\section{Conflicts of Interest}

The biospecimens and data used for this study were provided by the Biobank of Pusan National University Hospital, a member of the Korea Biobank Network.

\section{Research Funding}

None declared.

\section{ORCID}

Kyung-Hwa Shin https://orcid.org/0000-0002-8454-4448 Hyung-Hoi Kim https://orcid.org/0000-0003-3989-2037 Byung Su Kwon https://orcid.org/0000-0002-9586-0200 Dong Soo Suh https://orcid.org/0000-0001-5785-4355 Jong Kil Joo https://orcid.org/0000-0002-6338-1512 Ki Hyung Kim https://orcid.org/0000-0003-2364-5875

\section{REFERENCES}

1. Bray F, Ferlay J, Soerjomataram I, Siegel RL, Torre LA, Jemal A. Global cancer statistics 2018: GLOBOCAN estimates of incidence and mortality worldwide for 36 cancers in 185 countries. CA Cancer J Clin 2018;68: 394-424.

2. Tchagang AB, Tewfik AH, DeRycke MS, Skubitz KM, Skubitz AP. Early detection of ovarian cancer using group biomarkers. Mol Cancer Ther 2008; 7:27-37.

3. Daly MB, Pilarski R, Berry MP, Buys SS, Friedman S, Garber JE, et al. NCCN Guidelines Insights: genetic/familial high-risk assessment: breast and ovarian, version 2.2017. J Natl Compr Canc Netw 2017;15:9-20.

4. Fotopoulou C, Hall M, Cruickshank D, Gabra H, Ganesan R, Hughes C, et al. British Gynaecological Cancer Society (BGCS) epithelial ovarian/ Fallopian tube/primary peritoneal cancer guidelines: recommendations for practice. Eur J Obstet Gynecol Reprod Biol 2017;213:123-39.

5. Committee on Gynecologic Practice, Society of Gynecologic Oncology. 
Committee Opinion No. 716. Committee Opinion No. 716: the role of the obstetrician-gynecologist in the early detection of epithelial ovarian cancer in women at average risk. Obstet Gynecol 2017;130:e146-9.

6. Escudero JM, Auge JM, Filella X, Torne A, Pahisa J, and Molina R. Comparison of serum human epididymis protein 4 with cancer antigen 125 as a tumor marker in patients with malignant and nonmalignant diseases. Clin Chem 2011;57:1534-44.

7. Aggarwal $P$ and Kehoe SJM. Serum tumour markers in gynaecological cancers. Maturitas 2010;67:46-53.

8. Schummer M, Ng WV, Bumgarner RE, Nelson PS, Schummer B, Bednarski DW, et al. Comparative hybridization of an array of 21,500 ovarian cDNAs for the discovery of genes overexpressed in ovarian carcinomas. Gene 1999;238:375-85.

9. Guadagni F, Roselli M, Cosimelli M, Ferroni P, Spila A, Cavaliere F, et al. CA 72-4 serum marker-a new tool in the management of carcinoma patients. Cancer Invest 1995;13:227-38.

10. Lenhard MS, Nehring S, Nagel D, Mayr D, Kirschenhofer A, Hertlein L, et al. Predictive value of CA 125 and CA 72-4 in ovarian borderline tumors. Clin Chem Lab Med 2009;47:537-42.

11. Granato T, Midulla C, Longo F, Colaprisca B, Frati L, Anastasi E. Role of HE4, CA72.4, and CA125 in monitoring ovarian cancer. Tumour Biol 2012;33:1335-9.

12. Yang WL, Gentry-Maharaj A, Simmons A, Ryan A, Fourkala EO, Lu Z, et al. Elevation of TP53 autoantibody before CA125 in preclinical invasive epithelial ovarian cancer. Clin Cancer Res 2017;23:5912-22.

13. Chudecka-Głaz AM. ROMA, an algorithm for ovarian cancer. Clin Chim Acta 2015;440:143-51.

14. Prat J and FIGO Committee on Gynecologic Oncology. FIGO's staging classification for cancer of the ovary, fallopian tube, and peritoneum: abridged republication. J Gynecol Oncol 2015;26:87-9.

15. Moore RG, Miller MC, Disilvestro P, Landrum LM, Gajewski W, Ball JJ, et al. Evaluation of the diagnostic accuracy of the risk of ovarian malignancy algorithm in women with a pelvic mass. Obstet Gynecol 2011;118: 280-8.

16. Ferraro S, Braga F, Lanzoni M, Boracchi P, Biganzoli EM, Panteghini M. Serum human epididymis protein 4 vs carbohydrate antigen 125 for ovarian cancer diagnosis: a systematic review. J Clin Pathol 2013;66:27381.

17. Wang J, Gao J, Yao H, Wu Z, Wang M, Qi J. Diagnostic accuracy of serum HE4, CA125 and ROMA in patients with ovarian cancer: a metaanalysis. Tumour Biol 2014;35:6127-38.

18. Scaletta G, Plotti F, Luvero D, Capriglione S, Montera R, Miranda A, et al. The role of novel biomarker HE4 in the diagnosis, prognosis and fol- low-up of ovarian cancer: a systematic review. Expert Rev Anticancer Ther 2017; 17:827-39.

19. Moore RG, McMeekin DS, Brown AK, DiSilvestro P, Miller MC, Allard WJ, et al. A novel multiple marker bioassay utilizing HE4 and CA125 for the prediction of ovarian cancer in patients with a pelvic mass. Gynecol Oncol 2009;112:40-6.

20. Bandiera E, Romani C, Specchia C, Zanotti L, Galli C, Ruggeri G, et al. Serum human epididymis protein 4 and risk for ovarian malignancy algorithm as new diagnostic and prognostic tools for epithelial ovarian management. Cancer Epidemiol Biomark Prev 2011;20:2496-506.

21. Ławicki S, Będkowska GE, Gacuta-Szumarska E, Szmitkowski M. The plasma concentration of VEGF, HE4 and CA125 as a new biomarkers panel in different stages and sub-types of epithelial ovarian tumors. J Ovarian Res 2013;6:45.

22. Van Gorp T, Cadron I, Despierre E, Daemen A, Leunen K, Amant F, et al. HE4 and CA125 as a diagnostic test in ovarian cancer: prospective validation of the Risk of Ovarian Malignancy Algorithm. Br J Cancer 2011; 104:863-70.

23. Moore RG, Miller MC, Steinhoff MM, Skates SJ, Lu KH, Lambert-Messerlian G, et al. Serum HE4 levels are less frequently elevated than CA125 in women with benign gynecologic disorders. Am J Obstet Gynecol 2012; 206:351.e1-8.

24. Anastasi E, Granato T, Falzarano R, Storelli P, Ticino A, Frati L, et al. The use of HE4, CA125 and CA72-4 biomarkers for differential diagnosis between ovarian endometrioma and epithelial ovarian cancer. J Ovarian Res 2013;6:44.

25. Nagy B Jr, Krasznai ZT, Balla H, Csobán M, Antal-Szalmás P, Hernádi Z, et al. Elevated human epididymis protein 4 concentrations in chronic kidney disease. Ann Clin Biochem 2012;49:377-80.

26. Moore RG, Brown AK, Miller MC, Skates S, Allard WJ, Verch T, et al. The use of multiple novel tumor biomarkers for the detection of ovarian carcinoma in patients with a pelvic mass. Gynecol Oncol 2008;108:402-8.

27. Jacob F, Meier M, Caduff R, Goldstein D, Pochechueva T, Hacker N, et al. No benefit from combining HE4 and CA 125 as ovarian tumor markers in a clinical setting. Gynecol Oncol 2011;121:487-91.

28. Chan KK, Chen CA, Nam JH, Ochiai K, Wilailak S, Choon AT, et al. The use of HE4 in the prediction of ovarian cancer in Asian women with a pelvic mass. Gynecol Oncol 2013;128:239-44.

29. Kadija S, Stefanovic A, Jeremic K, Radojevic MM, Nikolic L, Markovic I, et al. The utility of human epididymal protein 4 , cancer antigen 125 , and risk for malignancy algorithm in ovarian cancer and endometriosis. Int J Gynecol Cancer 2012;22:238-44. 